Témoigner Témoigner. Entre histoire et mémoire

Getuigen Revue pluridisciplinaire de la Fondation Auschwitz

$115 \mid 2013$

L'Espagne en construction mémorielle

\title{
La transmission et la construction de l'espace transgénérationnel
}

Transmission and construction of the transgenerational space

Overdracht en opbouw van de transgenerationele ruimte

Marta Marín-Dòmine

Traducteur : David Martens

\section{(2) OpenEdition}

1 Journals

Édition électronique

URL : http://journals.openedition.org/temoigner/470

DOI : 10.4000/temoigner.470

ISSN : 2506-6390

Éditeur :

Éditions du Centre d'études et de documentation Mémoire d'Auschwitz, Éditions Kimé

Édition imprimée

Date de publication : 1 mars 2013

Pagination : 97-107

ISBN : 978-2-84174-628-6

ISSN : 2031-4183

Référence électronique

Marta Marín-Dòmine, « La transmission et la construction de l'espace transgénérationnel », Témoigner. Entre histoire et mémoire [En ligne], 115 | 2013, mis en ligne le 01 juin 2015, consulté le 23 octobre 2020. URL : http://journals.openedition.org/temoigner/470 ; DOI : https://doi.org/10.4000/temoigner 470

Tous droits réservés 


\section{La transmission et la construction de l'espace transgénérationnel ${ }^{*}$}

MARTA MARÍN-DÒMINE

Centre for Memory and Testimony Studies,

Laurier University, Waterloo-Toronto

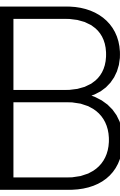

arcelone, mars 2008. Il a été admis à l'hôpital. Insuffisance cardiaque. J'ai attendu seule pendant des heures alors qu'on s'occupait de lui aux urgences. Lorsque j’ai pu entrer, je l'ai trouvé assis sur un lit, ou plutôt une civière, étroite et inconfortable, située dans le fond de la pièce, en partie voilée par un rideau qui la séparait de la civière d'à côté ainsi que d'autres dizaines de civières gémissantes et plaintives. Il régnait là une forte odeur de corps à laquelle je m'habituerai les jours suivants et à laquelle, d'une certaine manière, je suis reconnaissante. La vérité toute nue. Une vérité qu'il vaut mieux accepter sans broncher.

Avant même de manifester sa joie de me voir, il me fait un geste de la main pour m'indiquer qu'il veut me dire quelque chose de confidentiel. Quelque chose qui m'effraie sans que je sache ce dont il s'agit. Le pacte sera le suivant : il me fait promettre que je ne donnerai pas mon accord si les médecins suggèrent une intervention à cour ouvert. Un instant marqué par l'éventualité de la mort et à travers lequel un héritage symbolique s'établit.

Les jours passent. Il me raconte des anecdotes de la vie à l'hôpital ; la nuit dernière, me dit-il, une infirmière l'a grondé, comme s'il s'agissait d'un enfant, parce qu'il ne restait pas tranquille dans son lit et qu'il activait à chaque mouvement l'alarme de la machine à laquelle il était relié, réveillant ainsi tout le personnel. Il a répondu qu'il n'y était pour rien, que c'était eux qui l'avaient attaché à cette chose. Et il a ajouté : «D'ailleurs, ne me parlez pas sur ce ton! Moi, j’ai fait la guerre. » Je le connais, je sais qu'il l'a sans doute dit avec ironie, peut-être l'une des dernières ressources dont les

(*) Cet article a été rédigé à partir d'une conférence présentée au 4 e Congres International El Exilio Republicano de 1939 y la Segunda Generación, Bellaterra (15-18 décembre) et Collioure (19 décembre), Grupo de Estudios del Exilio Literario (GEXEL), Université Autònoma de Barcelone, 2009 et fait partie du projet de recherche HAR2011-2349 du Ministère de Science et Compétitivité, Espagne. 
vieux de ce pays disposent pour montrer qu'ils existent par le biais de la rhétorique. Toutefois, l'infirmière a rétorqué : «Ah ouais ? Et vous l'avez gagnée ${ }^{1}$ ? »

Ce que je prends pour du cynisme dans sa réponse me pousse à toute une série de réflexions. Je m'interroge alors sur les possibles positions d'un individu vis-à-vis des événements du passé et de leur éventuelle transmission : quels sont, me demandé-je, les éléments en jeu, les différences éthiques entre un individu qui décide de s'ouvrir au passé, et par conséquent à la différence, d'un autre qui décide de le refuser en l'ignorant de son propre chef, ce qui n'est en fin de compte qu'une forme de cynisme?

Même si c'est par extrapolation, la réaction de l'infirmière pourrait servir à illustrer le paradigme éthique d'une société, la société espagnole en l'occurrence, toujours dans un processus d'autodéfinition, sur la base des paramètres d'une modernité construite à partir de l'intronisation d'un présupposé universel (rêverie utopique à la recherche du même) qui efface la valeur de l'expérience subjective malgré l'utilisation de discours en faveur du multi - ou du pluri-, et tout cela peut nous laisser croire que la prétendue différence est acceptée, voire officiellement bien reçue. Cependant, dans le cas des « vaincus », la différence qui se construit marginalement est dévalorisée, ignorée. Le cynisme vis-à-vis de l'expérience d'autrui n'est donc pas surprenant, surtout lorsque l'on considère que cet autre n'a « rien » apporté à l'histoire officielle.

Donner une valeur aux événements sur la base de ce qu'ils comportent d'universalisable me conduit à poser la question suivante : l'universalisable ne serat-il pas l'un des obstacles de la transmission du passé, puisque ce qui est en jeu dans la transmission, c'est la circulation de l'expérience en tant que subjectivité et, par conséquent, l'affirmation du témoignage comme différence, acceptant ce qu'il y a de variable et de multiple dans chaque témoignage lié à l'événement en question ${ }^{2}$ ?

Et la seconde : comment transmettre ce qui est resté gravé dans la mémoire du témoin, « témoin » comme « spectateur» de l'événement et non victime de celui-ci, mais dont le message peine à s'inscrire dans l'histoire?

J'ai parfois le sentiment que l'une des dimensions de la transmission entre générations est la reconstruction de l'expérience - qui ne peut être autre chose que sa représentation - tenant compte des symptômes qui sont restés gravés sur le corps - le corps du témoin, mais aussi le corps de celui à qui l'expérience est racontée. Des corps en tant que réceptacles, des corps en tant que représentation.

Cette dimension est celle que Wajdi Mouawad, dramaturge canadien d'origine libanaise, présente à travers ses œuvres. Dans son théâtre, la référence à l'impact sensoriel de la guerre sur les survivants est constante. Un impact que montrent les

(1) En effet, mon père appartenait à ce que l'on considère comme le camp vaincu par l'armée fasciste des troupes du général Francisco Franco en 1939.

(2) J'entends ici par « universel » la demande de totalité que l'on exige souvent des récits testimoniaux. L'exigence de récits globalisateurs et, par conséquent, représentatifs de la « généralité » pourrait être considérée comme un élément clef dans la construction d'un effet de «saturation » mémorielle, alors que l'on refuse le subjectif et le particulier et que l'on privilégie le général et l'universel. Ainsi, toute narration particulière, aussi nouvelle soit-elle, est perçue comme un excès donné qui s'ajoute à ce qui est censé être connu, c'est-à-dire le général qui efface la particularité subjective de n'importe quel récit. 
pièces de théâtre au moyen d'une dramaturgie qui met les corps en contact, corps virtuels des survivants, corps réels des acteurs et des spectateurs. Au sujet des premières neiges qu'il a connues au Québec, lieu d'exil de l'auteur, Mouawad raconte que ce fut face à la vision des rues de cette ville, salies par le dégel, qu'il a ressenti à nouveau cette inquiétude qui le rongeait lorsqu'il était enfant et qu'il jouait au milieu des décombres couverts de boue de sa ville natale défigurée par les bombardements ${ }^{3}$. Une expérience où le témoin et les lieux sont donc métonymiquement liés par des traits du passé gravés sur le corps, des marques qui donnent forme à la relation des témoins avec le monde, avec les gens, avec les lieux.

C'est dans le cadre de l'hommage à ce que l'on appelle les « deuxième et troisième générations » que je m'aventure pour la première fois à formuler les réflexions suivantes. Je veux dire par là que je me base sur la position que je m'octroie en tant que témoin de «troisième génération » et en qualité de réceptrice directe de l'expérience de la deuxième génération - celle des « enfants de la guerre civile espagnole ». Un acte qui est aussi une forme de reconnaissance, un hommage.

Selon moi, « rendre hommage » implique de se situer dans la dynamique de la transmission, même si pour pouvoir insuffler ce mouvement auquel elle contraint nous devons nous préserver d'une mythification pétrifiante qui reste aveugle aux avatars de l'histoire et barre l'accès à l'analyse objective des causes et des conséquences des événements. Elle nous immobilise. J'utilise dès lors cet espace pour lancer des questions - pour «bouger» - à un moment historique que je qualifierais de critique puisque la transmission, dans son versant espagnol, prise à partir du syntagme « récupération de la mémoire historique », s'articule à mi-chemin entre les effets de l'occultation exercés par un long silence et la prolifération des commémorations officielles.

\section{TRANSMETTRE, TÉMOIGNER}

La transmission, qui s'opère la plupart du temps par la filiation, ne devrait-elle pas plutôt se définir à partir du moment où un individu accepte le passé en tant qu'héritage? Cette acceptation n'est-elle pas l'indice de sa participation à la représentation du passé, puisque la transmission comporte implicitement la recréation, l'interprétation, la modification du témoignage ${ }^{4}$ ? C'est ainsi que le passé survit, sans être limité à la possession des histoires de famille, circulant comme un objet qui rend possible le lien social, sans toutefois ignorer l'impact que l'événement a eu dans le privé, dans le cercle familial. Le passé, donc, en tant que trace qui permet par le biais du témoignage sa conversion en une histoire commun(autair)e. Élargir la frontière du vécu, permettre,

(3) Wajdi Mouawad, « Wajdi Mouawad, enfant dans la guerre, exilé sans frontières », propos recueillis par Brigitte Salino, entretien publié dans Le Monde du 7 juillet 2009. Disponible en ligne sur : http://www.lemonde. fr/culture/article/2009/07/07/wajdi-mouawad-enfant-dans-la-guerre-exile-sans-frontieres_1215695_3246. html

(4) Comme exemple de cette conceptualisation de l'héritage, je vous invite à consulter mon article : Marta Marín-Dòmine, Sueños y pesadillas: los efectos subjetivos de la Guerra Civil española a través de la mirada cinematográfica, in Amnis, Université de Bretagne occidentale, 2006, p. 269-284. 
comme dans l'anecdote de Wajdi Mouawad, que l'expérience entre dans une relation dynamique avec d'autres temps et d'autres lieux. Intégrer l'unique, la différence, au commun du tissu sociétal.

C'est cette perspective qui me permet de réclamer en tant qu'héritage l'expérience vécue par mon père, sans que je veuille pour autant me l'approprier. Transmettre, c'est à la fois accepter et donner. Reconnaître l'expérience de l'autre en tant que telle, sans la posséder.

\section{JOAQUIM MARÍN CABALLOL, UN ENFANT QUI A VÉCU PENDANT LA GUERRE}

Mon hommage à la deuxième génération de l'exil républicain dans son ensemble est construit sur l'expérience d'un enfant, aujourd'hui mon père, qui est passé par deux espaces géographiques distincts et qui, par conséquent, a expérimenté la difficulté de vivre et de transmettre l'expérience d'une façon que l'on évite habituellement lorsque l’on fait référence à l'« exil » ou aux effets de la répression franquiste sur les individus. Une brève biographie s'impose :

Joaquim Marín Caballol (né en 1924) vit à Barcelone, dans ce qui était un quartier ouvrier dynamique, celui de Sant Martí de Provençals ; celui-ci comptait de nombreux « cercles ». Au milieu du XIX ${ }^{\mathrm{e}}$ siècle, le quartier était connu comme le « Manchester catalan » à cause du grand nombre de fabriques textiles qu'il abritait. Les « cercles » et les autres associations tenaient lieu d'écoles pour les enfants le jour et pour les adultes la nuit, l'objectif premier étant l'éducation des ouvriers et des ouvrières.

Le quartier de Sant Martí de Provençals a subi de nombreuses transformations, toutes très agressives, comme la construction de l'avenue Meridiana, une artère très fréquentée qui divise le quartier en deux au détriment de la vie sociale qui s'y était développée. Aujourd'hui, cette vie associative reprend vie grâce à l'action de divers groupes de jeunes, dont certains, et le lien avec la question de la transmission et des conséquences de la récupération de l'histoire occultée est direct, montrent un intérêt réellement surprenant envers l'histoire du quartier qu'ils veulent connaître et faire connaître et qui abrite aujourd'hui un nombre important de nouveaux immigrés. Un exemple curieux est celui d'un groupe de jeunes appartenant au mouvement okupa qui, il y a quelques années dans un édifice abandonné et récupéré au profit du quartier, a créé le centre « La Revoltosa » où sont proposées des activités culturelles, un petit service de restauration ainsi que des cours de formation à l' idéologie anarchiste.

Je me promène dans le quartier en offrant mon bras à Joaquim, qui m'indique du doigt le lieu exact où les gens du quartier, majoritairement des femmes et des enfants, ont construit un abri antiaérien, une activité à laquelle il a lui aussi contribué. À côté de cette invisibilité - aucun panneau n'indique ni n'indiquera cet abri - les okupas ont aujourd'hui aménagé un jardin potager biologique qu'ils ont mis au service du quartier. Des actions sociales qui se mêlent et se projettent dans l'histoire.

Le père de Joaquim, qui portait le même nom, était surnommé dans le quartier $E l$ Panxo de Sant Martí. Ouvrier dans la construction, de filiation anarchiste, il a participé 
activement aux révoltes ouvrières des années 1920, à tel point qu'il dut s'exiler en 1929 sur la recommandation de son avocat qui craignait, après trois incarcérations, que son client soit touché par les actions violentes du patronat.

Ce n'était pas le premier exil de Joaquim « Panxo ». Il avait déjà été chassé au Maroc, mais c'était la première fois qu'il avait été suivi, jusqu’à Béziers, par toute sa famille - sa femme, son fils et sa fille. Son fils venait d'avoir cinq ans et l'instant qui précéda le départ a été immortalisé par une photographie prise à Barcelone, que j’ai fait agrandir lorsque j’ai commencé à travailler dans une imprimerie autogérée à l'École d'ingénieurs de Barcelone. Sur cette photo, on peut voir un enfant inquiet ; ses yeux, toutefois, n'ont encore pratiquement rien vu.

La mère de cet enfant meurt à Béziers et la famille rentre à Barcelone en 1935. Pendant cette période, celui qui sera mon grand-père poursuit son compromis politique et, lors de la révolution, en 1936 (c'est sous ce nom que l'événement politique est évoqué par ma famille paternelle), le Syndicat le désigne « responsable ouvrier dans une fabrique collective » à Barcelone puisqu'il n'était pas appelé sous les drapeaux à cause de son âge. Joaquim fils raconte que son père composait des discours enflammés que lui, un enfant de douze ans à l'époque, était chargé de copier sous la dictée. «Écris, lui ordonnait son père, écris : “Comme le disait Kropotkine...” » Mon grand-père prononçait Kropotkine en laissant flotter le « e » final, comme suspendu dans l'air, à la française. Un « e » dont je me souviens encore et qui apparaissait souvent dans les mots que lui et mon père s'échangeaient : le monde fascinant et étranger des « e » errants. Les discours écrits par Joaquim fils étaient ensuite lus dans les fabriques, harangues écrites dont le but était d'encourager les ouvriers dans leur production révolutionnaire.

En 1937, l'enfant, grâce aux contacts de son père dans l'aviation, du côté de la localité catalane de Sabadell, s'enrôle dans le corps des aviateurs. Trop jeune pour être soldat, le capitaine lui propose alors de faire partie de la clique. Joaquim, insatisfait par cette perspective, parvient à être chargé de l'économat du camp et, par conséquent, de la répartition des repas (de la «gamelle») des soldats. Joaquim écrit dans ses mémoires:

Ce jour-là, on m’a fait commencer les cours de tambour dans un coin du camp d'aviation avec d'autres jeunes camarades. Une infinie tristesse m'envahit, je ne sais pas pourquoi... Peut-être était-ce parce que je voyais mon rêve brisé... Moi, j’avais voulu être enrôlé comme soldat et non comme joueur de tambour. Je pensais pressentir que j’étais destiné à autre chose, que javais un meilleur niveau que mes camarades qui semblaient s'amuser en jouant des instruments du groupe. Moi, au contraire, je craignais ce pauvre destin... J'ai tout de suite accepté d'être chargé de l'économat du camp5.

Lorsque Barcelone est tombée entre les mains des troupes franquistes, en janvier 1939, Joaquim fils entame, avec les officiers du corps d'aviation de Sabadell, le chemin de la Retirada: Sabadell, Granollers, Montmeló, Olot, Figueres, La Jonquera,

(5) Extrait des mémoires inédites de Joaquim Marín Caballol. 


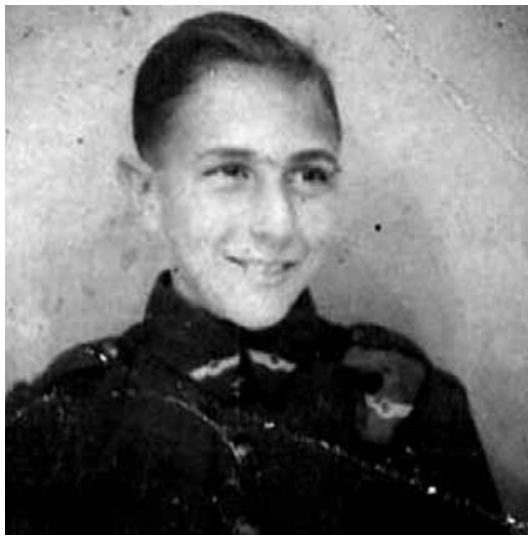

Joaquim Marín Caballol, à son entrée dans le corps d'aviation de Sabadell, 1938. (๑) Archive personnelle.
Le Boulou, le camp de concentration d'Argelès et le camp de concentration de Saint-Cyprien. De Figueres, il garde le souvenir du bombardement des avions fascistes. Joaquim prend le chemin de l'exil en compagnie d'un autre jeune camarade, Trueba, le cornettiste du camp d'aviation de Sabadell. Avant de passer la frontière, les deux enfants, lors d'une cérémonie privée, enterrent le cornet sous un olivier dont ils notent les coordonnées - ce sont des lecteurs de Jules Verne. Ils passent la frontière avec l'intention de revenir et se disent que, sur le chemin du retour, ces paramètres leur permettront de déterrer le cornet.

Une fois la frontière traversée, ces enfants exigent d'être acceptés comme soldats de l'armée républicaine et suivent la marche des hommes vers Argelès, tout d'abord, et vers Saint-Cyprien ensuite pour y être intégrés. Plus tard, ils seront rapatriés en Espagne, via Irún. Pour atteindre Barcelone, ils devront entreprendre un périple semé d'embûches rendues inévitables par le parcours auquel les fascistes les obligent: les deux jeunes, qui allaient être envoyés dans une arène faisant office de camp de concentration, parviennent à séchapper, avant d'être découverts et envoyés dans un couvent de religieuses à Saragosse, pour racheter les fautes des « rouges», institution qu'ils réussissent à fuir également. Ils finiront par atteindre Barcelone et retrouver leurs familles.

L'enfant, deuxième génération de la guerre et de l'exil, appartient à ce petit groupe de préadolescents qui a vécu l'exil dans les camps et du très petit nombre quil l'a vécu en qualité de très jeune soldat. De très jeunes soldats superbement photographiés par Manuel Moros, bien évidemment. C'est aussi le paradigme de ceux qui sont revenus, sous la contrainte, et qui ont apporté l'expérience d'un exil frustré, d'un aller suspendu, l'impression d'avoir laissé échapper une vie où la vie aurait été possible.

Pour moi, témoin de "troisième génération », l'expérience de mon père peut être représentée par un long murmure soutenu : des murmures en passant devant certains bâtiments ou paysages, des murmures en mentionnant la chance de certains voisins, exilés en France. Des murmures épouvantés en évoquant la mort dans les camps nazis de l'un ou l'autre parent. Je m'interroge, par conséquent, sur les effets du passé d'autrui dans ma perception de l'espace au présent.

J'écris ces lignes depuis le Canada où je vis. Métonymie de lieux qui traverse les générations : depuis le « e » suspendu jusqu’à la phrase prononcée dans un lieu où elle ne peut être écoutée. Des espaces qui s'ajoutent, sans solution de continuité, à la chaîne transgénérationnelle des exils, certains - comme le mien - par choix, d'autres non. Je devrais sans doute réaliser une étude sur le caractère insupportable, pour la troisième génération, des lieux marqués par l'exil familial. Une génération en 
mouvement, que ce soit pour rencontrer les lieux abandonnés par les parents et les grands-parents ou pour s'en éloigner. Une dialectique particulière qui se dirige vers l'inconnu et s'éloigne de l'excès de ce qui représente la famille.

\section{SOUVENIRS ET FLASH-BACK}

Joaquim, dans ses souvenirs, mais aussi et toujours à travers ses lectures, a vécu comme tant d'autres avec les yeux rivés sur la France ; avec l'angoisse provoquée par la tension entre l'affection que l'on est censé avoir pour ses origines et le désir d'y renoncer. Mon père me raconte qu'il ne sent pas Barcelone comme étant sienne, qu'il ne l'a jamais sentie sienne depuis 1939. « Ils nous l'ont volée », me dit-il. Et il le dit au téléphone, lui à Barcelone, moi à Paris, au cours d'un de mes séjours plus ou moins errants entre l'Amérique et l'Europe. Ses mots m'attristent profondément, mais ils me donnent le courage de continuer à errer - errer est aussi synonyme de se tromper. Me trompé-je vraiment dans mon choix d'autres lieux, toujours d'autres lieux?

Barcelone, peut-être, 1971. Je me souviens vaguement du moment où mon père m'a raconté qu'il s'était retrouvé, dans son enfance, à l'âge de quatorze ans, dans un camp de concentration ${ }^{6}$. Moi, je sais déjà ce qu'est un « camp de concentration ». Toutefois, à la maison, où aucun livre n'est jamais interdit, l'ouvrage intitulé La déportation était proscrit'. Ce livre montrait, sur la couverture, la terrible photographie du visage d'un survivant des camps nazis. Impossible. Obscène, diront mes parents. La souffrance d'autrui doit être reconnue, sans être dévoilée. Ce livre, par conséquent, ne pouvait franchir le seuil de la maison. Je parviendrai néanmoins à y jeter un œil, chez mon amie Cristina, et nous l'avons fait ensemble en cachette, nous sentant à la fois fascinées et sales.

Lorsque mon père m’a raconté qu'il était allé dans un camp de concentration, jai ressenti une étrange sensation de torpeur, comme si ma vision se troublait. Il me racontait que les camps français, ceux du Sud de la France (toujours avec l'article devant, cette France, la France de son enfance), étaient différents. Il se hâtait ensuite de diminuer l'importance de son expérience ; ma peur l'effrayait. Il me parlait de la faim et du froid. Mon père m'en transmettait le souvenir par sa crainte récurrente de me voir attraper une pneumonie à chaque fois que j'étais mouillée par la pluie. Des préoccupations présentes dans d'autres familles espagnoles et qui ont parsemé l'expérience infantile d'autres enfants d'hommes et de femmes qui sont passés par les camps français ${ }^{8}$.

(6) Cf. les discours répétés d’Albert Sarraut, ministre français de l'Intérieur, en 1939, et que le journal La Dépêche a publié en février de la même année : « Le camp d’Argelès-sur-Mer ne sera pas un lieu pénitentiaire mais un camp de concentration. Ce n'est pas la même chose. Les asilés qui y prendront séjour n'y resteront guère que le temps nécessaire pour préparer leur refoulement ou, sur leur option, leur libre passage de retour en Espagne. »

(7) Leroy Andre Alcan, Louise Almann Jean, La Déportation, Le Patriote Résistant FNDIRP, 1967.

(8) Cf., par exemple, Gisèle Matamala, Cette Lettre oubliée, Paris, Les Passés Simples, 2004. 


\section{BRÈVES RÉFLEXIONS (WORK-IN-PROGRESS)}

Aline Angoustures raconte dans une œuvre collective ${ }^{9}$ les difficultés rencontrées par les adultes espagnols réfugiés en France lorsqu'ils tentent de transmettre à leurs enfants l'expérience de la guerre et de l'exil. L'une des composantes essentielles de ces difficultés, souligne-t-elle, sont les problèmes dérivés de l'énonciation ; soit parce que les parents - adultes pendant la guerre, souvent avec le poids de l'expérience des camps de concentration français sur les épaules - ont été incapables de parler, soit parce que l'excès de propos a rendu l'écoute insupportable pour leurs enfants.

Il convient ici de signaler que les enfants auxquels elle se réfère sont pour la plupart nés en France. Dans ces circonstances, revivre le passé de leurs parents agit comme un frein à leur intégration dans un pays qui est déjà le leur, même s'il reste « le pays étranger » pour les parents. Ainsi, le passé est évoqué comme une période figée, presque comme un présent en suspension qui fait obstacle, pour les enfants, tant à l'appropriation imaginaire du pays où ils sont nés qu'à l'identification ou à l'intérêt, et par conséquent à l'expérience, vis-à-vis du pays de leurs parents.

Ailleurs dans le même ouvrage, Florence Guilhem séquence la transmission de l'expérience des adultes espagnols en France et leur plus ou moins grande difficulté à s'exprimer en étapes chronologiques qui sont effectivement marquées par un éventail complexe de charges émotionnelles : la guerre civile, l'exode en France, le passage par les camps, la vie sous l'occupation... Des récits qui s'accompagnent le plus souvent de la transmission parallèle de positions idéologiques - toutes celles de gauche - dont la fonction symbolique est de justifier, vis-à-vis d'eux-mêmes et de leurs enfants, l'exil ${ }^{10}$. Cependant, la réception du témoignage de cette expérience est toujours conflictuelle pour les enfants puisqu'elle renferme la perception d'un héroïsme qui est dans une certaine mesure démenti par l'image - de douleur, ou de nostalgie, du père ou de la mère qui témoigne - de l'un des parents vaincus dans un pays étranger dans lequel l'assimilation est souvent complexe. C'est cette situation que les enfants évitent, situation clef dans la transmission de l'expérience.

Ce sont donc des souvenirs qui se construisent en l'absence de l'espace qui leur a donné naissance. Ils sont, par conséquent, déterritorialisés (question : la mémoire n'estelle pas toujours déterritorialisée en exil ?), c'est la raison pour laquelle la construction imaginaire du pays d'origine se construit souvent à partir de la nostalgie ou de la mythification.

Pour Florence Guilhem, la fonction de la mémoire transmise de génération en génération, en exil, est de légitimer ce que la première génération a vécu, de sorte que la deuxième génération puisse avoir des éléments de référence pour justifier son existence dans le pays étranger des parents, ce qui lui permettra de considérer

(9) Aline Angoustures, «Transmissions familiales chez des enfants de réfugiés politiques espagnols en France », in Enfants de la Guerre civile espagnole. Vécus et représentations de la génération née entre 1925 et 1940 , Paris, L'Harmattan, 1999, p. 109-122.

(10) Florence Guilhem, « D'une Guerre à l'autre ; mémoire des pères, histoire des fils », in Ibid., p. 123-138. 
l'expérience de la première génération comme partie intégrante de son identité en devenir : un imbroglio toujours positif en ce qu'il implique d'ouverture à la différence, à la multiplicité, à l'incertain, à l'inattendu présent en autrui et dans l'individu lui-même.

Finalement, le point sur lequel les études d'Aline Angoustures et de Florence Guilhem - dont les analyses sont bien plus complexes que ce que mes brefs commentaires laissent entrevoir - concordent, c'est que, malgré les difficultés de la première génération à transmettre et de la deuxième génération à écouter, la transmission a bien eu lieu, en particulier dans ces zones géographiques où la proportion de réfugiés espagnols a été significative, permettant ainsi un partage collectif des idéaux politiques et l'évitement du sentiment d'isolement. La majeure partie des personnes interrogées par Florence Guilhem, des habitants de la région de Toulouse, se trouve dans cette situation. Elle utilise la métaphore d'un « fond sonore familial », une musique toujours présente ${ }^{\mathrm{ll}}$, pour se référer à la mémoire de l'exil : il revient donc à chacun de se l'approprier, de l'écouter ou de l'ignorer.

Le cas de la transmission et de la réception de l'expérience de la guerre civile dans la deuxième génération qui a grandi en Espagne, sous la pression de la dictature, est différent. On sait, et c'est une preuve de la reconnaissance tardive de ce passé en Espagne, que la transmission intergénérationnelle n’a pas seulement été difficile pour les raisons qui concernent les Espagnols en exil, mais aussi parce que la « musique de fond » qui, en Espagne, aurait rendu possible la reconnaissance du passé partagé en son sein n’a jamais existé. Le murmure en revanche - et nous devrions ici reconsidérer et problématiser la métaphore du silence pour faire référence au passé censuré - est l'élément qui est venu se substituer à la possibilité du fond sonore. Un silence sonore qu'il faudra distinguer de l'oubli, concept que l'on a trop précipitamment diffusé pour justifier les difficultés de la mémorisation et de la transmission du passé des vaincus.

En effet, considérer le silence - silence imposé et par conséquent admis et transmis - comme une difficulté majeure de la transmission mémorielle à l'intérieur du pays implique d'ouvrir l'analyse au politique dans sa relation avec l'expérience subjective.

Nous pourrions donc dire que la transmission du vécu, durant la période historique qui nous occupe, 1936-1945, est marquée par les absences : absence évidente du territoire géographique de remémoration dans le cas de l'exil consécutifà la guerre; absence, également, sur le territoire espagnol de circulation des témoignages de l'expérience des camps français, pas uniquement à cause de l'éloignement et de l'exil, mais aussi à cause de la censure imposée à l'intérieur du pays.

\section{ESPACE OUVERT À L'INTERROGATION}

Permettez-moi de terminer par des questions qui font déjà l'objet de réflexions et qui visent à en induire d'autres à l'avenir. Il est évident que l'espace géographique

(11) Ibid., p. 137. 
mémoriel qui semble, jusqu'à aujourd'hui, s'être détaché lors de la construction de l'expérience vécue durant l'exil, est celui qui correspond logiquement à l'expérience vécue dans les pays d'accueil ou de transit. Cependant, il y a tout un espace à préserver, celui des témoignages - dans ses formes diverses, tant orales qu'écrites ou picturales réalisés sur le territoire espagnol par des exilés qui l'ont été de manière très provisoire, qui sont revenus la même année, en 1939, et qui sont demeurés dans un murmure silencieux, toujours par crainte d'être l'objet de représailles. Il est par conséquent impératif d'appliquer une méthodologie qui permette d'élucider les diverses formes de modalité de transmission de l'événement, à partir du lieu dans lequel il se produit (l'exil ou l'« intérieur », dans leurs diverses modalités) et, plus significatif encore, à partir du lieu imaginaire d'où ils sont énoncés. La distinction, dans ce cas, entre l'énoncé et le lieu de l'énonciation nous permet d'aller plus loin que la traditionnelle séparation entre « intérieur » et « extérieur ». Il s'agirait de percevoir le témoin de l'expérience comme un individu changeant et variable qui peu à peu construit le lieu, à partir de son expérience passée et présente et selon les effets qu'il souhaite obtenir de ce témoignage. Raconter un événement aux enfants, à la jeune génération, ou l'écrire pour un lecteur inconnu, par exemple, sont deux choses différentes.

Tenir compte du lieu de l'énonciation permet également de prendre conscience que l'exil n'a pas supposé l'érection de barrières infranchissables, que les gens ont bougé, ont déménagé, sont revenus dans le pays d'origine lors de visites sporadiques ou périodiques, etc., et que les constructions de l'expérience, par conséquent, dépassent la dichotomie restrictive que constituent l'exil et l'« intérieur »; et, très important, qu'il existe également une multiplicité de constructions d'espaces imaginaires, qui comprennent certainement ceux construits sur la nostalgie, mais aussi d'autres construits sur l'utopie.

En ce qui concerne mon père - son cas constitue le fil d'Ariane dans les méandres de ces réflexions -, son deuxième exil et son retour au pays ont généré en lui un sentiment d'exclusion permanent, de telle sorte que le témoignage de l'expérience transmise supposa quelques fois la possibilité imaginaire qu'il soit dehors, dans l'espace rêvé, et d'autres fois lui permit de constater qu'il était resté sans espace. L'espace imaginaire idéal a été « cet autre lieu » qui avait un nom concret, la France, mais qui était à son tour un espace imaginaire et imaginé : l'espace d'un premier exil, de son école française, républicaine et laïque ; l'espace de la frontière, vers lequel nous revenions timidement, dans une Renault des années 1960, et qui me semblait respirer et vivre. La vie était, simplement, de l'autre côté de la frontière.

Ainsi, de la même manière que, pour des raisons humaines, psychologiques, historiques et sociologiques évidentes, des études ont été réalisées pour analyser l'impact que l'expérience des immigrants espagnols a eu sur leurs enfants, il conviendrait également d'analyser l'impact de l'expérience des enfants d'Espagnols qui ont transmigré ou qui sont restés immergés dans l'État répressif, entre deux eaux. Il est certain que la littérature nous offre des exemples multiples de représentation d'un après-guerre fatidique, mais dans lesquels la représentation de l'exil - les camps, les centres de détention - n'a pas pu voir le jour (à la suite d'un mélange d'ignorance 
et de censure). C'est maintenant, bien que timidement, que commence à circuler l'expérience issue de la construction littéraire ${ }^{12}$.

Enfin, comment intégrer dans le présent les murmures qui ont transporté les corps ayant vécu une expérience réduite au silence? Comment rendre visible, comme le raconte le philosophe Jacques Rancière, l'histoire de ceux qui ont été maintenus dans l'ombre ? Comment transmettre l'expérience sans qu'elle semble rance, pauvre, faible, innocente ? Comment construire une musique de fond qui rende possible le passage de l'individuel au collectif?

L'écriture, sans aucun doute, est une possibilité. Mais, à nouveau, une question surgit : à quelle perspective éthique a-t-on recours lorsque l'on sollicite la mémoire de l'autre ? Parce que, comme je l'ai déjà mentionné au début de cet article, une seule question suffit pour nous positionner en tant que transmetteurs ou en tant qu'ignorants du passé. Et de cela, nous sommes responsables. Pas seulement «les enfants de », mais les « générations » qui témoignent de la vie, en filiation avec la communauté humaine.

Traduit de l'espagnol par David Martens

(12) À ce sujet, on peut citer les ouvrages suivants : Miguel Catalán, El último Juan Balaguer, Valence, Algar, 2002 ; Jordi Soler, Los rojos de ultramar, Barcelone, Alfaguara, 2004 [Les Exilés de la mémoire, traduction JeanMarie Saint-Lu, Paris, Belfond, 2007] ; Lluís-Anton Baulenas, Per un sac d'ossos, Barcelone, Planeta, 2005. 